\title{
Video Article \\ Measurement of Coherence Decay in GaMnAs Using Femtosecond Four-wave Mixing
}

\author{
Daniel Webber ${ }^{1}$, Tristan de Boer ${ }^{1}$, Murat Yildirim ${ }^{1}$, Sam March $^{1}$, Reuble Mathew ${ }^{1}$, Angela Gamouras ${ }^{1}$, Xinyu Liư ${ }^{2}$, Margaret Dobrowolska ${ }^{2}$, \\ Jacek Furdyna ${ }^{2}$, Kimberley Hall ${ }^{1}$ \\ ${ }^{1}$ Department of Physics and Atmospheric Science, Dalhousie University \\ ${ }^{2}$ Department of Physics, University of Notre Dame
}

Correspondence to: Kimberley Hall at km730924@dal.ca

URL: https://www.jove.com/video/51094

DOI: doi:10.3791/51094

Keywords: Physics, Issue 82, Four-wave mixing, spin-flip scattering, ultrafast, GaMnAs, diluted magnetic semiconductor, photon echo, dephasing, GaAs, low temperature grown semiconductor, exchange, ferromagnetic

Date Published: 12/3/2013

Citation: Webber, D., de Boer, T., Yildirim, M., March, S., Mathew, R., Gamouras, A., Liu, X., Dobrowolska, M., Furdyna, J., Hall, K. Measurement of Coherence Decay in GaMnAs Using Femtosecond Four-wave Mixing. J. Vis. Exp. (82), e51094, doi:10.3791/51094 (2013).

\section{Abstract}

The application of femtosecond four-wave mixing to the study of fundamental properties of diluted magnetic semiconductors ((s,p)-d hybridization, spin-flip scattering) is described, using experiments on GaMnAs as a prototype III-Mn-V system. Spectrally-resolved and timeresolved experimental configurations are described, including the use of zero-background autocorrelation techniques for pulse optimization. The etching process used to prepare GaMnAs samples for four-wave mixing experiments is also highlighted. The high temporal resolution of this technique, afforded by the use of short ( $20 \mathrm{fsec}$ ) optical pulses, permits the rapid spin-flip scattering process in this system to be studied directly in the time domain, providing new insight into the strong exchange coupling responsible for carrier-mediated ferromagnetism. We also show that spectral resolution of the four-wave mixing signal allows one to extract clear signatures of (s,p)-d hybridization in this system, unlike linear spectroscopy techniques. This increased sensitivity is due to the nonlinearity of the technique, which suppresses defect-related contributions to the optical response. This method may be used to measure the time scale for coherence decay (tied to the fastest scattering processes) in a wide variety of semiconductor systems of interest for next generation electronics and optoelectronics.

\section{Video Link}

The video component of this article can be found at https://www.jove.com/video/51094/

\section{Introduction}

III-V diluted magnetic semiconductors exhibit ferromagnetic coupling mediated by carriers (holes), permitting external manipulation of magnetic characteristics using electrical and optical control of the carrier population. This feature makes these materials attractive for a whole host of magneto-sensitive electronic and optoelectronic devices ${ }^{1-15}$, including the possible integration of logic and memory. ${ }^{11-15}$ Realizing such applications requires a good understanding of the fundamental properties of these materials; however, the large density of defects associated with their growth at low temperatures (essential for the substitutional incorporation of magnetic dopants such as Mn) severely complicates the theoretical treatment of exchange coupling and ferromagnetic order ${ }^{16-18}$. These defects also impede the interpretation of transport and linear optical experiments on these materials ${ }^{19-24}$.

The utility of the nonlinear optical technique of four-wave mixing spectroscopy for gaining new insight into these materials was recently revealed in experiments on GaMnAs ${ }^{25,26}$, representing the prototype III-V diluted magnetic semiconductor. The nonlinearity of the technique was shown to provide a much greater sensitivity to fine structure in the optical joint density of states, indicating a strong enhancement of the interband optical response with the incorporation of $\mathrm{Mn}^{25}$. This observation is a signature of $(\mathrm{s}, \mathrm{p})$-d hybridization that had been recently predicted in tight-binding calculations $^{18}$. This new insight was afforded by the low optical dipole moment associated with transitions involving the mid-gap defect states, which mask these effects of hybridization in linear optical experiments ${ }^{27}$. The high time-resolution of the four-wave mixing technique has also been used to obtain the first direct measurement of the time-scale of hole-Mn spin flip scattering in this system ${ }^{26}$, providing new insight into exchange coupling. These experiments also showed that the optical response of this material is a photon echo, indicating that the dephasing time is independent of carrier energy despite defect-induced localization.

Here we present a detailed description of the four-wave mixing technique used in references ${ }^{25,26}$, illustrating how it may be used to measure the dephasing time (T2), the nonlinear (third-order) spectral response, and the nature of broadening (homogeneous or inhomogeneous) in a material of interest. Spectrally-resolved and temporally-resolved experimental configurations are featured, together with an explanation of the zerobackground autocorrelation technique used in conjunction with a prism compressor to minimize the pulse duration at the sample position. This pulse duration determines the overall time-resolution of the technique and must be below 30 fsec to resolve spin-flip scattering in GaMnAs. The etching process used to prepare the GaMnAs samples for experiments in the transmission geometry is also highlighted. This work builds upon the established utility of four-wave mixing techniques for studying scattering processes and many-body effects in traditional semiconductors ${ }^{28-32}$ 
by extending it to the investigation of fundamental interactions and electronic structure in diluted magnetic semiconductors. Our results will have implications for the long-term engineering of these novel magnetic materials.

\section{Overview of Optical Apparatus}

1. Set up two mirrors and two irises defining the input path for the optical setup, as shown in Figure 1. Each day, these irises and mirrors should be used to recover the setup alignment. Since the laser drifts day to day, several iterations of alignment are required on a daily basis to maintain optimal alignment.

2. Construct a pulse compressor containing two prisms, as shown in Figure 1. The pulse compressor introduces negative group velocity dispersion to compensate for the positive group velocity dispersion caused by lenses and optical windows on the way to the sample. The amount of group velocity dispersion compensation is determined by the separation between the prisms (coarse) and the amount of glass the beam propagates through in the second prism (fine). These are iteratively adjusted to optimize the pulse duration while detecting the pulse autocorrelation (See section 2.)

3. Insert a 50/50 beam splitter to divide the incoming pulses from the laser into two excitation pulses, E1 and E2.

4. Set up an optical delay line for pulse E1 using a pair of mirrors mounted on a manual translation stage. This stage is used to make coarse adjustments of the delay between pulses E1 and E2. Note: one must ensure that the optical path into the mirror pair is parallel to the axis of motion of the translation stage and that the mirrors are aligned at $45^{\circ}$ to each other and do not introduce vertical deviation.

5. Set up an optical delay line for pulse E2 using a set of mirrors and an optical periscope that send the beam down onto a lightweight retroreflector mounted on the cone of a speaker. Mounting the speaker vertically minimizes anharmonic motion of the speaker caused by the weight of the retroreflector as well as deleterious movement (jiggle) of the retroreflector as the speaker moves.

Note: one must ensure that the beam path into the retroreflector is parallel to the axis of motion of the speaker (i.e. vertical). Together with a function generator and current amplifier, the speaker allows the inter-pulse delay to be rapidly scanned during the experiment. The current amplifier is needed to ensure a sufficiently-large amplitude of motion of the speaker $(>1 \mathrm{~mm})$. The linear portion of the speaker throw determines the total accessible pulse delay range in the experiment.

6. Ensure that the two optical beams are parallel, and insert a single lens (diameter $5 \mathrm{~cm}$, focal length $50 \mathrm{~cm}$ ) into the path of the two beams, as shown in Figure 1. This lens is used to focus the two beams onto the sample contained in an optical cryostat, allowing the sample to be held at any desired temperature between $4-300 \mathrm{~K}$

7. Spatial and temporal overlap at the sample position are optimized by performing an autocorrelation at an equivalent focus in preparation for detection of the four-wave mixing signal. (The autocorrelation alignment is described in section 2.).

8. For spectrally-resolved, time-integrated experiments (SR-FWM), send the four-wave mixing emission into a monochromator, adding a lens to focus into the input slit.

9. Place a high-speed photomultiplier tube detector (PMT) after the monochromator to detect the spectrally-resolved four-wave mixing emission Note: the high voltage applied to the PMT should only be turned on with the PMT in the dark.

10. Connect the PMT output signal to an oscilloscope.

11. Using a function generator and current amplifier, set the speaker to oscillate at approximately $30 \mathrm{~Hz}$ to introduce a periodic variation of the time delay between pulses E1 and E2. Perform a measurement of the SR-FWM signal (PMT signal) versus pulse delay using the oscilloscope. The calibration of the time axis of the oscilloscope is determined using the autocorrelation signal, as described in section 2.

12. For time-resolved four-wave mixing (TR-FWM), insert a second 50/50 beam splitter to create a third laser pulse (called the gate pulse), as shown in Figure 1.

13. Set up a separate optical delay line for the gate pulse. This delay line contains a set of mirrors mounted to a manual translation stage for coarse adjustment of the gate pulse delay, and a second speaker for rapid scanning of the gate pulse delay.

14. Remove the lens in front of the monochromator and insert a mirror to pick off the four-wave mixing beam.

15. Insert a lens to focus the four-wave mixing emission, together with the gate pulse, into a Beta Barium Borate (BBO) nonlinear crystal cut for type I phase matching for $800 \mathrm{~nm}$ at normal incidence. Perform a measurement of the second harmonic intensity as a function of gate pulse delay using another high-speed PMT.

16. Set up a path for autocorrelation measurements by inserting a large planar mirror in front of the cryostat. This mirror is used to redirect both E1 and E2 pulses to a second focus. Here, place a second BBO crystal. Note: the input beams must be blocked during movement of this planar mirror to avoid hazardous reflections.

17. Perform a measurement of the second harmonic signal resulting from the overlap of pulses $E 1$ and E2 as a function of the interpulse delay, which is scanned using the speaker in the path of pulse E2. The second harmonic signal is detected using a fast photodiode and the oscilloscope. During this measurement, the pulse compressor alignment is optimized to provide the minimum possible pulse duration, as described in more detail in section 2.

\section{Pulse Measurement and Optimization}

1. Insert a large planar mirror to redirect the focal point of the two excitation beams from the sample to the secondary focus, as shown in Figure 1.

2. Place the BBO crystal at the focal point. This is used to generate second harmonic from the combination of the two input pulses E1 and E2. The crystal holder must allow for adjustments along all three spatial axes as well as rotation.

3. Place a short-pass optical filter after the crystal that rejects $800 \mathrm{~nm}$ light and transmits $400 \mathrm{~nm}$ light.

4. Two violet beams should be visible on a white card placed after the filter. These beams correspond to the second harmonic from each pulse $\mathrm{E} 1$ and $\mathrm{E} 2$ alone. Set the angle of rotation of the crystal so that it is approximately half way between the angles giving the maximum brightness of each of the two outer spots. This will be the approximate angle that optimizes the zero-background autocorrelation beam from the second harmonic from the combination of the two input pulses E1 and E2. 
5. Adjust the path length of the $\mathrm{E} 1$ pulse until a central second harmonic spot appears. This spot is the zero-background autocorrelation resulting from the combination of E1 and E2.

6. Insert a fast silicon photodiode in the path of the central second harmonic spot.

7. Vary the path length of pulse E2 periodically by using a function generator and amplifier to control the motion of the speaker in the E2 path, as described in section 1.

8. Connect the photodiode to an oscilloscope and record the observed autocorrelation. To properly view the autocorrelation, trigger the oscilloscope on a TTL pulse from the function generator. The TTL trigger pulse may need to be delayed using a digital delay generator to allow the autocorrelation to be observed on a convenient time axis setting on the oscilloscope.

9. Adjust the path length of pulse E1 using the manual translation stage and note how this adjustment shifts the temporal position of the peak of the second harmonic signal on the oscilloscope. The recorded micrometer positions for two successive peak positions may be used to convert the oscilloscope time axis to pulse delay (in femtoseconds).

10. Adjust the positions of the two prisms in the pulse compressor while viewing the autocorrelation signal on the oscilloscope in order to ensure that the autocorrelation peak is as narrow as possible. The minimum pulse duration achieved in these experiments was approximately 20 fsec.

\section{Sample Preparation}

1. For our four-wave mixing experiments, the sample consisted of a $500 \mu \mathrm{m}$ GaAs substrate, a $175 \mathrm{~nm}$ AlGaAs stop etch layer and a $750 \mathrm{~nm}$ thin film of GaMnAs. This sample was grown by molecular beam epitaxy at the University of Notre Dame.

2. These four-wave mixing experiments are carried out in the transmission geometry, and as a result, the substrate must be removed. Place a small bead of optical adhesive onto a sapphire window. Sapphire provides an excellent substrate for experiments at low temperature due to its good transparency and large thermal conductivity. Place the sample with the GaMnAs thin film face down onto the optical adhesive and press it down uniformly to remove excess glue from beneath the sample surface.

3. Cure the optical adhesive by exposing the sample to an ultraviolet lamp for approximately $6 \mathrm{hr}$. The lamp radiation must expose the sample from the sapphire side to cure the adhesive.

4. Using a mechanical polishing/grinding system (e.g. Buehler Beta Grinder-Polisher), remove 300-400 $\mu \mathrm{m}$ from the substrate. This step substantially reduces the time required for chemical etching.

5. Remove the remaining substrate by immersing the sample in a solution of citric acid ( 5 parts $6.28 \mathrm{M}$ ) and hydrogen peroxide ( 1 part, $30 \%$ solution). When the stop etch layer is reached, the sample will transition from a dull grey to a mirror-like finish, indicating that it can be removed from the solution. The average etch rate for GaAs is $20 \mu \mathrm{m} / \mathrm{hr}$ and for AIGaAs is $160 \mathrm{~nm} / \mathrm{hr}$ for this solution.

6. Remove the sample from the acid solution and rinse in deionized water, followed by spectroscopic-grade acetone, and then methanol.

7. Mount the sample in the optical cryostat at the focal point of the E1 and E2 beams. Evacuate and cool the cryostat to the desired temperature.

\section{Measurement of the Four-wave Mixing Signal}

1. Once the autocorrelation signal is optimized, the sample has been mounted in the cryostat, and the cryostat has been cooled, remove the large flat mirror used to redirect the laser focus.

2. To perform SR-FWM experiments, focus the four-wave mixing signal beam into a monochromator. To locate the four-wave mixing signal beam, first locate the transmitted E1 and E2 spots on an alignment tool (with wavevectors $\mathbf{k} 1$ and $\mathbf{k 2}$ ) and use their positions to determine the expected position of the four-wave mixing signal beam (2k2 - $\mathbf{k} \mathbf{1})$.

3. Place a high speed PMT at the output of the monochromator and connect it to the oscilloscope. Note: the high voltage bias on the photomultiplier tube must only be turned on with the detector in the dark.

4. With the function generator and amplifier powered on, leading to a periodic motion of the speaker in the path of pulse E2, use data acquisition software to record the oscilloscope trace for each desired wavelength setting of the monochromator. This trace consists of the four-wave mixing signal versus the delay between pulses E1 and E2. The trace is averaged over several scans, the number of which being chosen using the digital oscilloscope.

Note: The dynamic range in this case is limited by the analog to digital converter in the oscilloscope. If detailed structure in the tail of the signal is desired, one can either increase the sensitivity of the voltage scale or acquire the four-wave mixing signal using a fast data acquisition board instead of an oscilloscope

5. To perform TR-FWM measurements, remove the lens focusing the beam into the monochromator and insert a mirror to pick off the four-wave mixing signal beam.

6. Focus the four-wave mixing beam and the gate pulse into a BBO crystal, followed by an optical filter which passes only the sum frequency beam.

7. Perform a measurement of the cross correlation of the signal and gate pulse using a PMT. Vary the gate pulse delay by connecting the function generator and amplifier to the speaker in the gate pulse path.

8. Record the TR-FWM signal by using data acquisition software to record the oscilloscope trace for fixed values of the delay between pulses $\mathrm{E} 1$ and E2, which can be set using the manual translation stage in the beam path for pulse E1.

\section{Representative Results}

Typical results of a SR-FWM experiment on GaxMn1-xAs $(x=0.005 \%)$ are shown in Figure 2(b), together with results on a reference sample of GaAs grown under the same low-temperature conditions (Figure 2(a)). The success of the experiments is indicated by the high signal to noise ratio, allowing variations in the signal amplitude (indicated by the color scale) with both time delay and photon energy to be clearly assessed. A high signal to noise ratio is achieved by carefully optimizing the sample position in order to locate a clear spot on the sample with minimal scattered light from the excitation pulses. A successful experiment also requires that the sample thickness be comparable to the absorption depth for the photon energy of the excitation pulses. This maximizes the optical signal from the sample without introducing propagation effects that would complicate the analysis. For the results in Figure 2, the optical density was 0.2 . The broadening of the SR-FWM signal towards high 
energies in GaMnAs relative to GaAs is attributed to the influence of (s,p)-d hybridization of the substitutional Mn within the GaAs host crystal on the valence band density of states ${ }^{25}$.

A slice of the two-dimensional SR-FWM data in Figure 2(b) is shown in Figure 3(a) for a fixed photon energy of $1.533 \mathrm{eV}$, illustrating the dependence of the signal on the delay between the excitation pulses. This delay-dependent signal may be fit using an analytic model ${ }^{33}$ convoluted with the finite laser pulse profile (measured using zero-background autocorrelation techniques), allowing the dephasing time for electron-hole pairs to be extracted ( $\mathrm{T} 2=65 \mathrm{fsec}$ for the data shown). These fits are indicated by the solid curves in Figure 3 . If many-body effects play a strong role in the sample under study, a more complicated analysis may be required ${ }^{34,35}$. This is the case for the SR-FWM data in low-temperature grown GaAs in Figure 2(a) at the exciton (1.515 eV), where the signal is mediated by exciton scattering with free carrier transitions ${ }^{34}$. In GaMnAs for energies above the band gap of GaAs, the simpler two-level model provides good agreement, indicating no evidence of many-body effects. Such effects contribute much more strongly in traditional GaAs grown at elevated temperatures ${ }^{25,26,35}$.

Results of TR-FWM experiments on the same GaMnAs sample are shown in Figure 3(b). For these data, the delay between the two excitation pulses is $54 \mathrm{fsec}$, and the horizontal axis shows the signal envelope versus the arrival time of the gate pulse relative to the four-wave mixing signal pulse at the BBO crystal. The position of the peak signal in TR-FWM experiments provides a way to distinguish between homogeneouslybroadened and inhomogeneously-broadened optical transitions, shown schematically in Figure 4. In the latter case, the simultaneous excitation of optical transitions with a range of resonance energies leads to a rephasing of the polarizations at all energies at a time $t \approx 2 t_{D}$, where $t$ is the time of arrival of the gate pulse relative to the four-wave mixing pulse and $t_{D}$ is the delay between pulses $E 1$ and $E 2$. Here $t=0$ is the arrival time of the pulse $E 1$. In Figure 3(b), $t_{D}=54 \mathrm{fsec}$, and the TR-FWM signal peaks at $t \approx 100 \mathrm{fsec}$, indicating a photon echo response for the interband optical transitions in this system. The TR-FWM signal for a range of values of the inter-pulse delay $t_{D}$ is shown in Figure 5 , indicating a shift of the signal to larger $t$ with increasing $t_{D}$. The excellent simultaneous agreement between the analytic model and both the SR-FWM (Figure 3(a)) and TR-FWM (Figure 3(b)) results indicates that many body effects do not contribute significantly and that the dephasing rate is independent of the transition energy of the electron-hole pairs, despite defect-induced localization in this system. From comparison with data in low-temperature-grown GaAs as well as GaAs grown at high temperature, the dominant dephasing process is identified as hole-Mn spin-flip scattering ${ }^{26}$, providing new insight into the exchange interaction responsible for ferromagnetic order in this system.

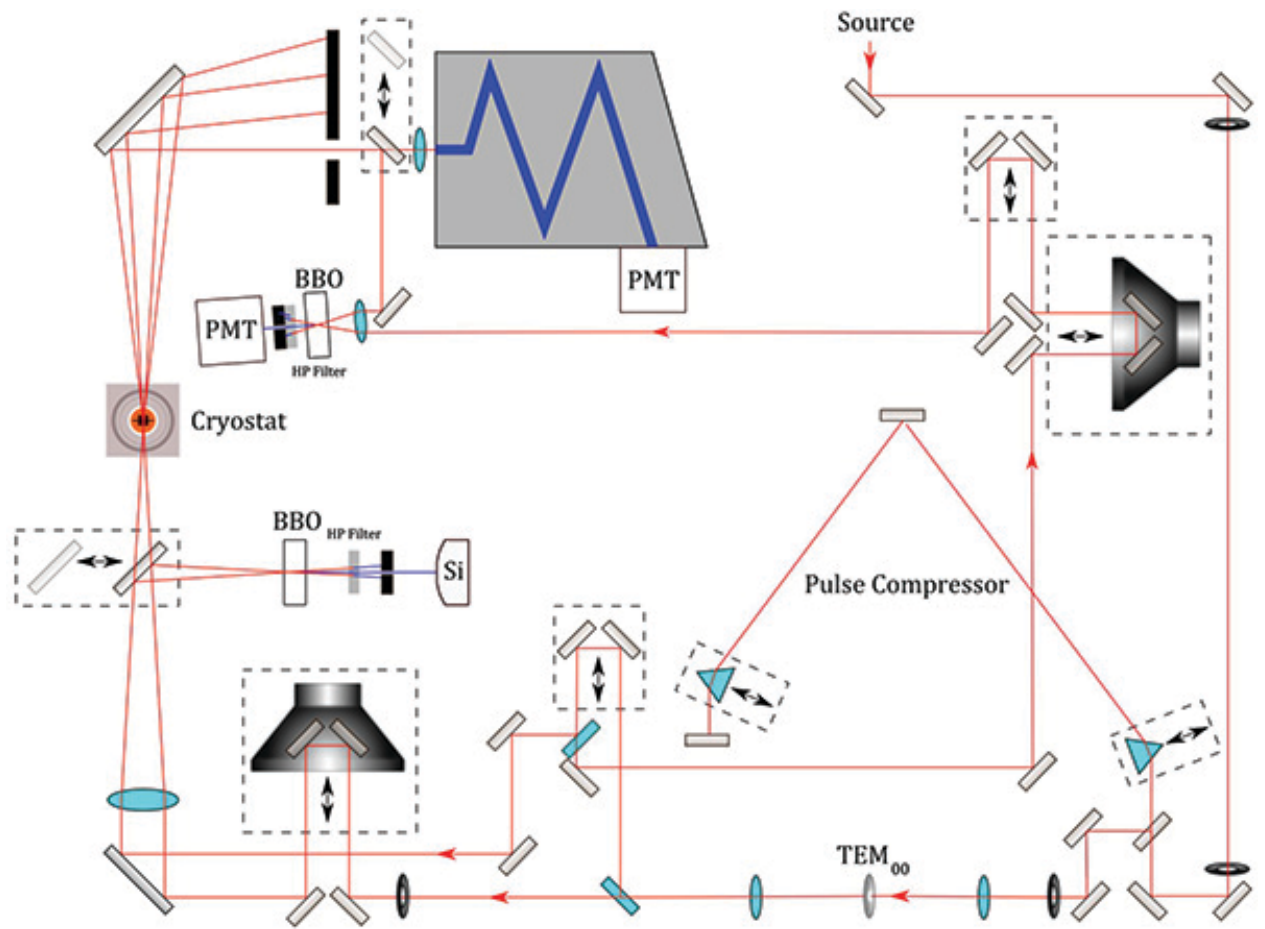

Figure 1. Schematic diagram of the Four-wave Mixing Apparatus. 

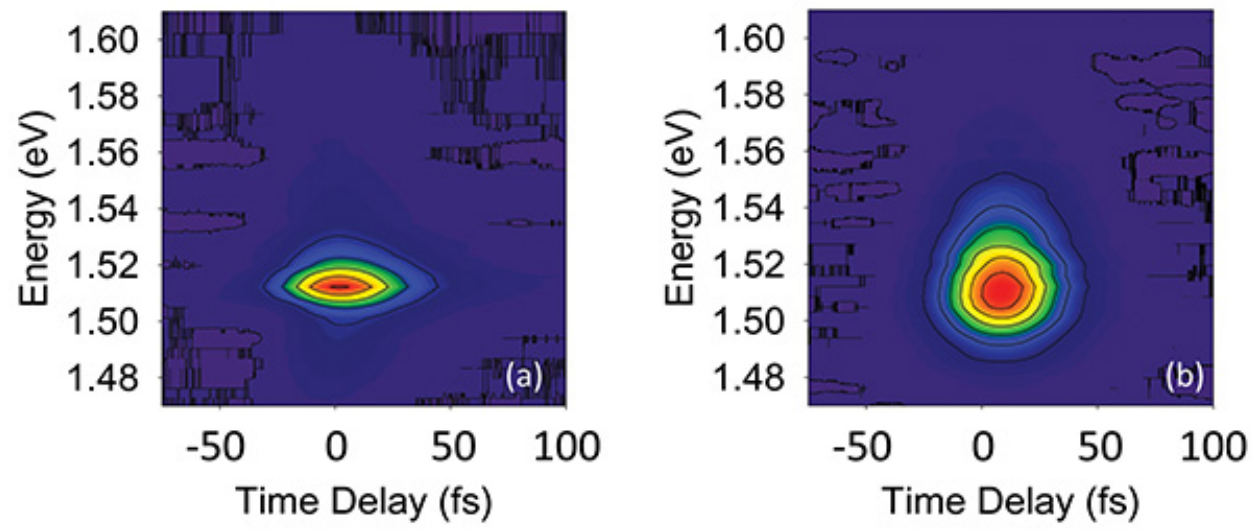

Figure 2. SR-FWM results from measurements on low-temperature-grown GaAs (left) and Ga1-xMnxAs for $x=0.005 \%$ (right). The vertical axis is the photon energy at which the signal is detected, selected using the monochromator, and the horizontal axis is the delay between pulses $\mathrm{E} 1$ and E2. All data shown were taken with the samples at $10 \mathrm{~K}$. Adapted with permission from reference ${ }^{25}$.
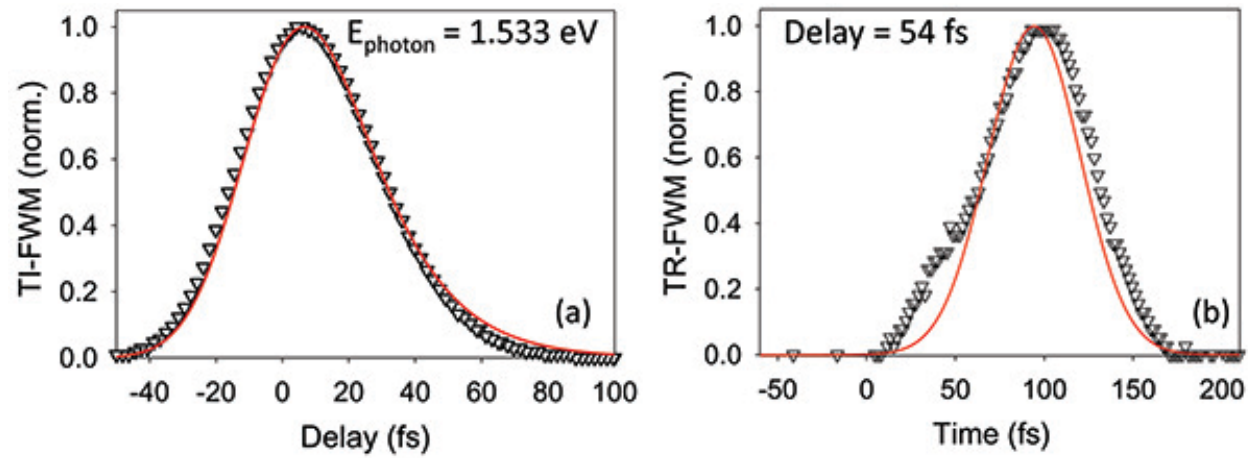

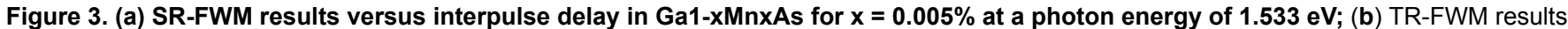
in the same sample versus the time of arrival of the gate pulse relative to the four-wave mixing pulse at the BBO crystal used for sum frequency generation, indicating the time envelope of the four-wave mixing pulse. For these data, the delay between pulses E1 and E2 is fixed at 54 fsec. The solid lines show fits using the analytic model in reference ${ }^{33}$. All data shown were taken with the sample at $10 \mathrm{~K}$. Part (b) adapted with permission from reference ${ }^{26}$.

\section{Homogeneous Broadening}

\section{Inhomogeneous Broadening}
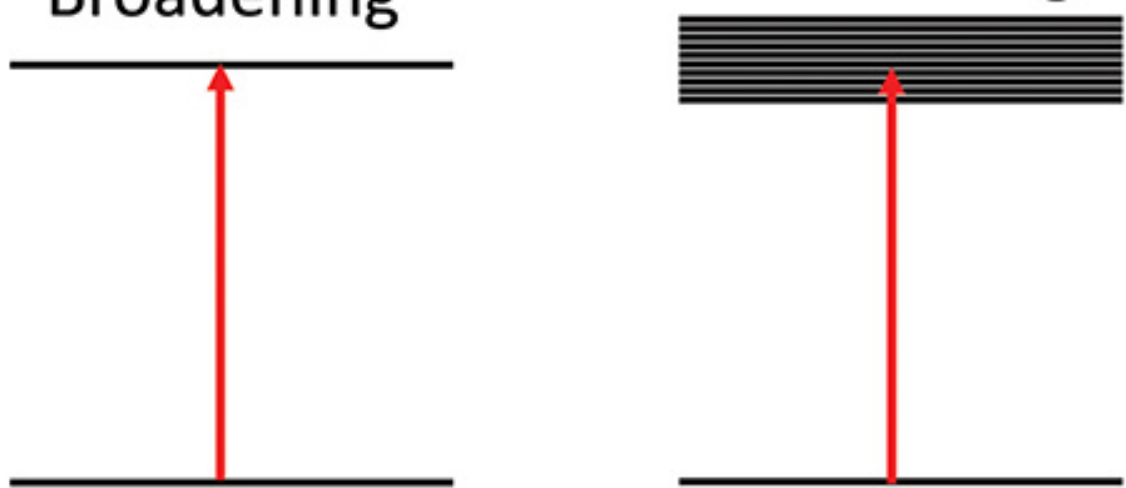

Figure 4. A schematic representation of a homogeneously-broadened (left) and inhomogeneously-broadened (right) two-level system. 


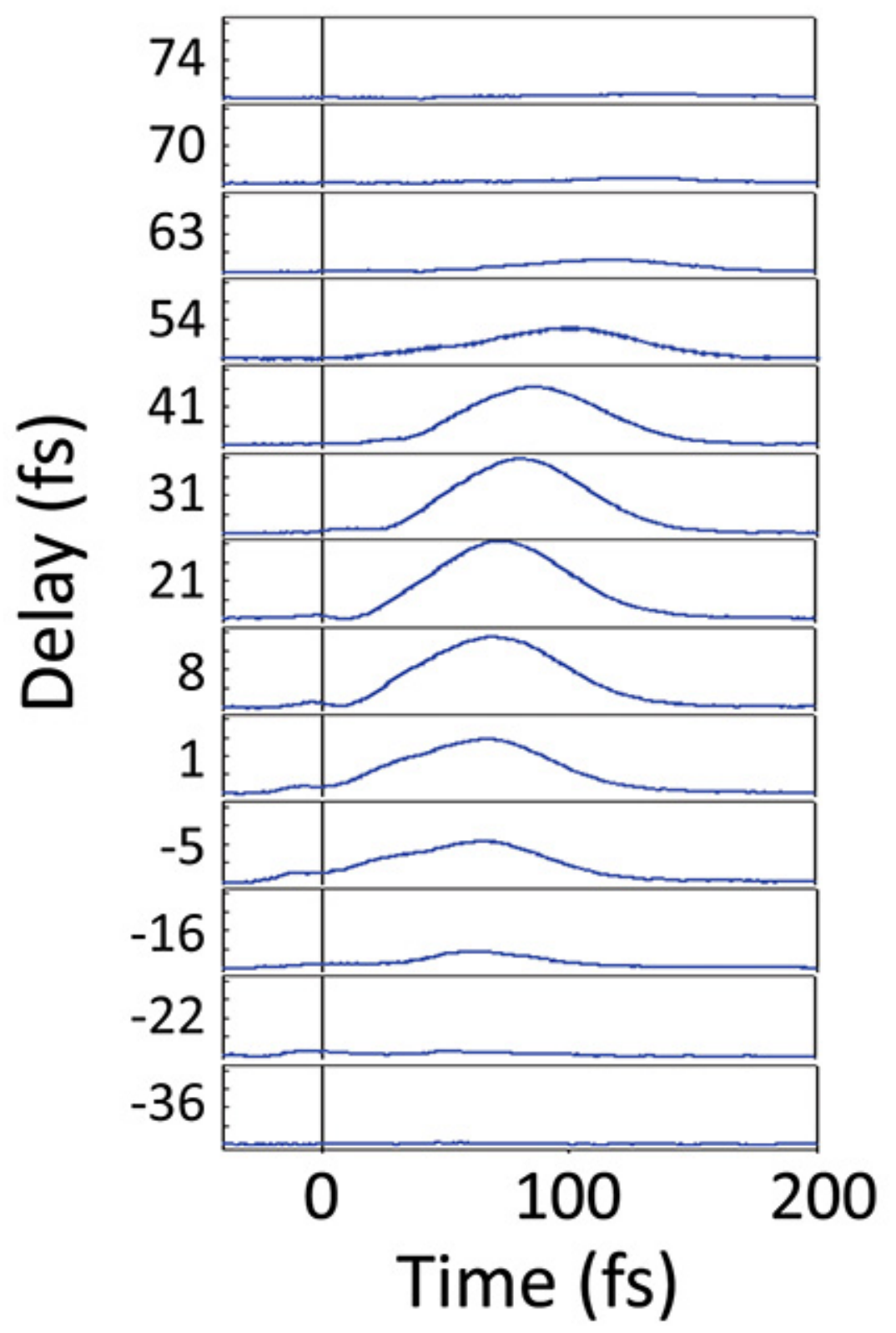

Figure 5. TR-FWM results for Ga1-xMnxAs for $x=0.005 \%$ as a function of the time of arrival of the gate pulse relative to the four-wave mixing pulse at the BB0 crystal used for sum-frequency generation. The signal is shown for various values of the delay between pulses $\mathrm{E} 1$ and $\mathrm{E} 2$, indicated on the vertical axis.

\section{Discussion}

The four-wave mixing technique may be used to measure the time scale for coherence decay on optical transitions in a wide range of material systems. For a variety of applications in advanced electronics and optoelectronics, this dephasing time is of crucial importance. For instance, in quantum computing applications (for which exciton transitions in semiconductors represent the fundamental quantum bit in several proposals ${ }^{36-38}$ ), the dephasing time determines the temporal window during which quantum operations and error correction must be carried out. The time scale for coherence decay is dictated by the fastest scattering processes of electrons and holes in the material, and so measurements of the dephasing time also provide insight into the nature and strength of the associated interactions responsible for these fast scattering processes. The type of interactions involved will depend strongly on the semiconductor system under study, and will govern both transport and coherence decay in any device application using this material. The four-wave mixing method therefore provides an important tool to characterize materials of interest for next generation electronic and optoelectronic devices. In such studies, the time-resolution is the most important limitation. This resolution is dictated by the optical pulse duration, which must be shorter than the dephasing time in the material under study. The specific experimental configuration described here is ideally applied to the study of fast decay processes (< a few picoseconds in 
duration). For measurement of slower temporal dynamics, lock-in detection methods and a slow-scanning delay stage with a larger range of motion are commonly employed.

Here we elucidate the utility of this technique for the investigation of III-V diluted magnetic semiconductors, in which strong hole-Mn exchange coupling leads to carrier-mediated ferromagnetism. Spin-flip scattering between holes and $\mathrm{Mn}$ ions is also caused by the hole-Mn exchange interaction. We have shown that four-wave mixing techniques may be used to obtain a direct measurement of the time scale of this process, providing insight into exchange coupling in this important class of materials. We have also shown that the nonlinearity of the four-wave mixing technique provides a highly sensitive probe of the band edge states in these materials, providing clear signatures of the (s,p)-d hybridization process that is the origin of the strong exchange coupling in this system. The increased sensitivity of four-wave mixing relative to linear optical techniques (e.g. absorption, ellipsometry, magnetic circular dichroism) to these band edge states stems from the nonlinearity of this technique, which suppresses defect-related contributions to the optical response.

This work lays the foundation for applications of this technique to study key physical properties of interest, such as the electronic structure and exchange coupling, in a wide range of III-V diluted magnetic semiconductors. This will allow these materials to be engineered for applications in semiconductor spintronics, including a whole host of magneto-sensitive electronic and optoelectronic devices.

\section{Disclosures}

The authors have nothing to disclose.

\section{Acknowledgements}

This research is supported by the Natural Sciences and Engineering Research Council of Canada, Lockheed Martin Corporation, and the National Science Foundation (Grant DMR10-05851).

\section{References}

1. Onodera, K., Masumoto, T. and Kimura, M. $980 \mathrm{~nm}$ compact optical isolators using Cd1-x-yMnxHgyTe single crystals for high power pumping laser diodes. Electron. Lett. 30, 1954-1955 (1994).

2. Kuroiwa, T., Yasuda, T., Matsukura, F,. Shen, A., Ohno, Y., Segawa, Y., and Ohno, H. Faraday rotation of ferromagnetic (Ga,Mn)As. Electron. Lett. 34, 190-192 (1998).

3. Ohya, S., Shimizu, H., Higo, Y., Sun, J., and Tanaka, M. Growth and Properties of Quaternary Alloy Magnetic Seminconductor (InGaMn)As. Jpn. J. Appl. Phys. Part 2. 41, L24-L27 (2002).

4. Wang, J., Sun, C., Kono, J., Oiwa, A., Munekata, H., Cywiński, L., and Sham., L.J. Ultrafast Quenching of Ferromagnetism in InMnAs Induced by Intense Laser Irradiation. Phys. Rev. Lett. 95, 167401-167405 (2005).

5. Zahn, J. P., Gamouras, A., March, S., Liu, X., Furdyna, J.K., and Hall., K.C. Ultrafast studies of carrier and magnetization dynamics in GaMnAs. J. Appl. Phys. 107, 033908-033917 (2010).

6. Rozkotova, E., Nemec, P., Tesarova, N., Maly, P., Novak, V., Olejnik, K., Cukr, M., and Jungwirth, T. Coherent control of magnetization precession in ferromagnetic semiconductor (Ga,Mn)As. Appl. Phys. Lett. 93, 232505-232508 (2008).

7. Astakhov, G.V., Kimel, A.V., Schott, G.M., Tsvetkov, A.A., Kirilyuk, A., Yakovlev, D.R., Karczewski, G., Ossau, W., Schmidt, G., Molenkamp, L.W., and Rasing. Th. Magnetization manipulation in (Ga,Mn)As by subpicosecond optical excitation. Appl. Phys. Lett. 86, 152506-152509 (2005).

8. Hall, K.C., Zahn, J.P., Gamouras, A., March, S., Robb, J.L., Liu, X., and Furdyna, J.K. Ultrafast optical control of coercivity in GaMnAs. Appl. Phys. Lett. 93, 032504-032507 (2008).

9. Reid, A.H.M., Astakhov, G.V., Kimel, A.V., Schott, G.M., Ossau, W., Brunner, K., Kirilyuk, A., Molenkamp, L.W., and Rasing, Th. Single picojoule pulse switching of magnetization in ferromagnetic (Ga,Mn)As. Appl. Phys. Lett. 97, 232503-232506 (2010).

10. Rudolph, J., Hägele, D., Gibbs, H.M., Khitrova, G., and Oestreich, M. Laser threshold reduction in a spintronic device. Appl. Phys. Lett. 82 4516-4519 (2003).

11. Wolf, S.A., Awschalom, D.D., Buhrman, R.A., Daughton, J.M., Molnar, S.von. Roukes, M.L., Chtchelkanova, A.Y., and Treger, D.M. Spintronics: A spin-based electronics vision for the future. Science. 294, 1488-1495 (2001).

12. Awschalom, D.D, Loss, D., and Samarth N. Semiconductor Spintronics and Quantum Computation. Springer-Verlag, Berlin (2002).

13. Datta, S., and Das, B. Electronic analog of the electro-optic modulator. Appl. Phys. Lett. 56, 665-667 (1990).

14. Hall, K.C., Lau, W.H., Gündoğdu, K., Flatté, M.E., and Boggess, T.F. Nonmagnetic semiconductor spin transistor. Appl. Phys. Lett. 83, 2937-2939 (2003).

15. Hall, K.C., and Flatté, M.E. Performance of a spin-based insulated gate field effect transistor. Appl. Phys. Lett. 88, 162503-162505 (2006).

16. Masek, J., Máca, D., Kudrnovsky, J., Makarovsky, O., Eaves, L., Campion, R.P., Edmonds, K.W., Rushforth, A.W., Foxon, C.T., Gallagher, B.L., Novák, V., Sinova, J., and Jungwirth, T. Microscopic analysis of the valence band and impurity band theories of (Ga,Mn)As. Phys. Rev. Lett. 105, 227202-227205 (2010).

17. Sheu, B.L., Myers, R.C., Tang, J.-M., Samarth, N. Awschalom, D.D., Schiffer, P., and Flatté, M.E. Onset of ferromagnetism in low-doped Ga1xMnxAs. Phys. Rev. Lett. 99, 227205-227208 (2007).

18. Tang, J.-M., and Flatté, M.E. Multiband tight-binding model of local magnetism in Ga1-xMnxAs. Phys. Rev. Lett. 92, 047201-047204 (2004).

19. Jungwirth, T., Horodyská, P., Tasarová, N., Nĕmec, P., Subrt, J., Malý, P., Kuzel, P., Kadlec, C., Masek, J., Nemec, I., M., Orlita, M., Novák, V., Olejník, K., Z. Sobáň, Z., Vasek, P., Svoboda, P., and Sinova, J. Systematic study of Mn-doping trends in optical properties of (Ga,Mn)As. Phys. Rev. Lett. 105, 227201 (2010).

20. Ohya, S., Takata, K., and Tanaka, M. Nearly non-magnetic valence band of the ferromagnetic seminconductor GaMnAs. Nature Phys. 7 , 342-347 (2011). 
21. Burch, K.S, Shrekenhamer, D.B., Singley, E.J., Stephens, J., Sheu, B.L., Kawakami, R.K., Schiffer, P., Samarth, N., Awschalom, D.D., and Basov, D.N. Impurity band conduction in a high temperature ferromagnetic semiconductor. Phys. Rev. Lett. 97, 087208-087211 (2006).

22. Acbas, G., Kim, M.-H., Cukr, M., Novák, V., Scarpulla, M.A., Dubon, O.D., Jungwirth, T., Sinova, J., and Cerne, J. Electronic structure of ferromagnetic semiconductor Ga1-xMnxAs probed by subgap magnetooptical spectroscopy. Phys. Rev. Lett. 103, 137201-137204 (2009).

23. Berciu, M., Chakarvorty, R., Zhou, Y.Y., Alam, M.T., Traudt, K., Jakiela, R., Barcz, A., Wojtowicz, T., Liu, X., Furdyna, J.K., and Dobrowolska, M. Origin of magnetic circular dichroism in GaMnAs: Giant Zeeman splitting versus spin dependent density of states. Phys. Rev. Lett. 102, 247202-247205 (2009).

24. de Boer, T., Gamouras, A., March, S., Novák, V., and Hall, K.C. Observation of a blue shift in the optical response at the fundamental band gap in Ga1-xMnxAs. Phys. Rev. B. 85, $033202-033206$ (2012).

25. Yildirim, M., March, S., Mathew, R., Gamouras, A., Liu, X., Dobrowolska, M., Furdyna, J.K., and Hall, K.C. Electronic structure of Ga1xMnxAs probed by four-wave mixing spectroscopy. Phys. Rev. B. 84, 121202(R)-121206(R) (2011).

26. Yildirim, M., March, S., Mathew, R., Gamouras, A., Liu, X., Dobrowolska, M., Furdyna, J.K., and Hall, K.C. Interband dephasing and photon echo response in GaMnAs. Appl. Phys. Lett. 101, 062403-062406 (2012).

27. Burch, K.S., Stephens, J., Kawakami, R.K., Awschalom, D.D., and Basov, D.N. Ellipsometric study of the electronic structure of Ga1-xMnxAs and low temperature GaAs. Phys. Rev. B. 70, 205208-205217 (2004).

28. 28. Kim, D.-S., Shah, J., Cunningham, J.E., Damen, T.C., Schäfer, W., Hartmann, M., and Schmitt-Rink, S. Giant excitonic resonance in timeresoled four-wave mixing in quantum wells. Phys. Rev. Lett. 68, 1006-1009 (1992).

29. Rappen, T., Peter, U., Wegener, M., and Schäfer, W. Coherent dynamics of continuum and exciton states studied by spectrally resolved fs four-wave mixing. Phys. Rev. B. 48, 4879-4882 (1993).

30. Cundiff, S.T., Koch, M., Knox, W.H., Shah, J., and Stolz, W. Optical coherence in semiconductors: Strong emission mediated by nondegenerate interactions. Phys. Rev. Lett. 77, 1107-1110 (1996).

31. Hall, K.C., Allan, G.R., van Driel, H.M., Krivosheeva, T., and Pötz, W. Coherent response of spin-orbit split-off excitons in InP: Isolation of many-body effects through interference. Phys. Rev. B. 65, 201201(R)-201204(R) (2002).

32. Shah, J. Ultrafast Spectroscopy of Semiconductors and Semiconductor Nanostructures. edited by Cardona, M. (Springer, Berlin) (1996).

33. Yajima, T., and Taira, Y. Spatial optical parametric coupling of picoseconds light pulses and transverse relaxation effect in resonant media. J. Phys. Soc. Jpn. 47, 1620-1626 (1979).

34. El Sayed, K., Birkedal, D., Lyssenko, V.G., and Hvam, J.M. Continuum contribution to excitonic four-wave mixing due to interaction-induced nonlinearities: A numerical study. Phys. Rev. B. 55, 2456-2465 (1997).

35. Hügel, W.A., Heinrich, M.F., Wegener, M., Vu, Q.T., Bányai, L., and Haug, H. Photon echoes from semiconductor band-to-band continuum transitions in the regime of coulomb quantum kinetics. Phys. Rev. Lett. 83, 3313-3316 (1999).

36. Reina, J.H., Quiroga, L., and Johnson, N.F. Quantum entanglement and information processing via excitons in optically driven quantum dots.Phys. Rev. A. 62, 012305-012312 (2000).

37. Troiani, F., Hohenester, U., and Molinari, E. Exploiting exciton-exciton interactions in semiconductor quantum dots for quantum-information processing. Phys. Rev. B. 62, R2263-R2266 (2000).

38. Biolatti, E., Lotti, R.C., Zanardi, P., and Rossi, F. Quantum information processing with semiconductors macroatoms. Phys. Rev. Lett. 85, 5647-5650 (2000). 